\section{(1) \\ CrossMark}

\title{
Pirfenidone safety and adverse event management in idiopathic pulmonary fibrosis
}

\author{
Lisa H. Lancaster ${ }^{1}$, Joao A. de Andrade ${ }^{2}$, Joseph D. Zibrak ${ }^{3}$, Maria L. Padilla ${ }^{4}$, \\ Carlo Albera ${ }^{5}$, Steven D. Nathan ${ }^{6}$, Marlies S. Wijsenbeek ${ }^{7}$, John L. Stauffer ${ }^{8}$, \\ Klaus-Uwe Kirchgaessler ${ }^{9}$ and Ulrich Costabel ${ }^{10}$
}

Affiliations: ${ }^{1}$ Vanderbilt University Medical Center, Nashville, TN, USA. ${ }^{2}$ University of Alabama at Birmingham, Birmingham, AL, USA. ${ }^{3}$ Beth Israel Deaconess Medical Center, Boston, MA, USA. ${ }^{4}$ Mount Sinai - National Jewish Health Respiratory Institute, New York, NY, USA. ${ }^{5}$ University of Turin, Turin, Italy. ${ }^{6}$ Inova Fairfax Hospital, Falls Church, VA, USA. ${ }^{7}$ Erasmus University Medical Centre Rotterdam, Rotterdam, The Netherlands. ${ }^{8}$ Genentech, Inc., South San Francisco, CA, USA. ${ }^{9} \mathrm{~F}$. Hoffmann-La Roche Ltd, Basel, Switzerland. ${ }^{10}$ Ruhrlandklinik, University of Duisberg-Essen, Essen, Germany.

Correspondence: Lisa H. Lancaster, Dept of Medicine, Vanderbilt University, Pulmonary and Critical Care, 1301 22nd Ave South, Nashville, TN 37232, USA. E-mail: lisa.lancasterवvanderbilt.edu

@ERSpublications

Using key strategies to prevent and manage pirfenidone-related AEs can help maximise adherence to pirfenidone http://ow.ly/Veyk30gsFTs

Cite this article as: Lancaster LH, de Andrade JA, Zibrak JD, et al. Pirfenidone safety and adverse event management in idiopathic pulmonary fibrosis. Eur Respir Rev 2017; 26: 170057 [https://doi.org/10.1183/ 16000617.0057-2017].

ABSTRACT Pirfenidone is one of two approved therapies for the treatment of idiopathic pulmonary fibrosis (IPF). Randomised controlled clinical trials and subsequent post hoc analyses have demonstrated that pirfenidone reduces lung function decline, decreases mortality and improves progression-free survival. Long-term extension trials, registries and real-world studies have also shown similar treatment effects with pirfenidone. However, for patients with IPF to obtain the maximum benefits of pirfenidone treatment, the potential adverse events (AEs) associated with pirfenidone need to be managed. This review highlights the well-known and established safety profile of pirfenidone based on randomised controlled clinical trials and real-world data. Key strategies for preventing and managing the most common pirfenidone-related AEs are described, with the goal of maximising adherence to pirfenidone with minimal AEs.

\section{Introduction}

Idiopathic pulmonary fibrosis (IPF) is a debilitating, progressive and fatal fibrotic lung disease, with an approximate median survival of 2-5 years from the time of diagnosis [1-4]. IPF is one of the most commonly encountered interstitial lung diseases (ILDs), with increasing incidence and prevalence worldwide [5-7].

Pirfenidone is an oral anti-fibrotic and anti-inflammatory agent that was previously approved in Europe in 2011 for the treatment of IPF and in the USA in 2014 [8]. In the 2015 update to the American Thoracic Society/European Respiratory Society/Japanese Respiratory Society/Latin American Thoracic Association

Received: May 122017 | Accepted after revision: Sept 072017

Support statement: Sponsorship for this publication was provided by F. Hoffmann-La Roche Ltd and Genentech, Inc. Support statements declared by individual authors can be found in the Acknowledgements section. Funding information for this article has been deposited with the Crossref Funder Registry.

Conflict of interest: Disclosures can be found alongside this article at err.ersjournals.com

Provenance: Submitted article, peer reviewed.

Copyright OERS 2017. ERR articles are open access and distributed under the terms of the Creative Commons Attribution Non-Commercial Licence 4.0. 
treatment guidelines, pirfenidone was listed as one of only two novel agents conditionally recommended for the treatment of IPF [9].

This review highlights the safety profile of pirfenidone based on clinical trial experience and real-world data. Strategies for mitigating the most common gastrointestinal (GI) and skin-related adverse events (AEs), as well as how to manage these AEs in patients with IPF based on clinical and real-world experience with pirfenidone, are described.

\section{Pirfenidone efficacy}

Pirfenidone was evaluated in patients with IPF in three pivotal, multinational, randomised, placebo-controlled phase III trials: CAPACITY (PIPF-004/006; NCT00287716/NCT00287729) and ASCEND (PIPF-016; NCT01366209) [10, 11]. The phase III clinical trials assessed pirfenidone in patients with IPF who had a forced vital capacity (FVC) of $\geqslant 50 \%$ predicted and diffusing capacity for carbon monoxide (DLCO) of $\geqslant 35 \%$ pred (CAPACITY) or FVC of $50-90 \%$ pred and DLCO of $\geqslant 30 \%$ pred (ASCEND). Improved outcomes, including reduced lung function decline, slowed disease progression and reduced mortality, were observed in patients treated with pirfenidone [10-13].

The benefit of continued treatment with pirfenidone despite disease progression was observed in pooled post hoc analyses from the phase III trials, emphasising the importance of persistence with pirfenidone treatment $[14,15]$.

In real-world studies of pirfenidone in patients with IPF, the effects of pirfenidone on reducing FVC decline were consistent with findings from the clinical trials [16-19]. However, differences in baseline characteristics and data analysis (including the availability of long-term follow-up data) may have affected how real-world data were interpreted. Of note, the inclusion criteria for clinical trials are very stringent and do not necessarily reflect real-world patients and practices.

\section{Pirfenidone safety and tolerability}

Pirfenidone has a well-characterised long-term safety and tolerability profile. The most commonly reported AEs due to pirfenidone are GI- and skin-related (table 1).

TABLE 1 Clinical and real-world safety experience with pirfenidone

\begin{tabular}{|c|c|c|c|c|}
\hline & \multicolumn{2}{|c|}{ Clinical trial experience } & \multicolumn{2}{|c|}{ Real-world experience } \\
\hline & $\begin{array}{l}\text { Pooled phase III } \\
\text { trials" }{ }^{\#}[12]\end{array}$ & $\begin{array}{c}\text { Integrated trials" } \\
{[20]}\end{array}$ & EAP [21] & PASSPORT [22] \\
\hline Patients $\mathrm{n}$ & 623 & 1299 & 1620 & 1009 \\
\hline Duration of treatment years ${ }^{+}$ & $1.0(0-2.3)$ & $1.7(0-9.9)$ & $0.45(0-0.91)$ & $1.0(0-2.4)$ \\
\hline Cumulative total exposure PEY & 735.7 & 3159.9 & 707.9 & 415 \\
\hline AE type & TEAE & TEAE & ADR & ADR \\
\hline \multicolumn{5}{|l|}{ TEAEs or ADRs per 100 PEY $\S$} \\
\hline Total & 418.8 & 790.2 & 419.6 & 67.0 \\
\hline Nausea & 38.6 & 26.0 & 62.1 & 20.4 \\
\hline Rash & 33.3 & 16.2 & 19.3 & 12.7 \\
\hline Diarrhoea & 26.0 & 20.1 & 27.6 & 10.6 \\
\hline Fatigue & 21.2 & 16.0 & 52.4 & 17.9 \\
\hline Dyspepsia & 16.3 & 9.5 & 16.4 & 4.6 \\
\hline Anorexia & 10.2 & 7.1 & 16.8 & NA \\
\hline Dizziness & 9.9 & 12.7 & 14.4 & 6.5 \\
\hline Gastro-oesophageal reflux disease & 7.1 & 6.6 & 12.7 & 1.9 \\
\hline Decreased appetite & 6.8 & 5.1 & 13.7 & 15.8 \\
\hline Decreased weight & 3.4 & 7.3 & 6.6 & 15.6 \\
\hline ADR/TEAE resulting in death & 2.2 & $18.5^{f}$ & NA & 1.4 \\
\hline ADR/TEAE leading to discontinuation & 14.6 & & 29.7 & 68.0 \\
\hline \multicolumn{5}{|c|}{$\begin{array}{l}\text { EAP: expanded access programme; PEY: patient-exposure years; AE: adverse event; TEAE: } \\
\text { treatment-emergent adverse event; ADR: adverse drug reaction; NA: not available. }{ }^{\#} \text { : CAPACITY (004/006) } \\
\text { and ASCEND (016); }{ }^{\uparrow}: \text { phase III trials+RECAP+PIPF-002, including two patients in the PIPF-002 study with a } \\
\text { diagnosis of "pulmonary fibrosis"; }{ }^{+}: \text {data presented as median (range); }{ }^{\S} \text { : one PEY is the equivalent of one } \\
\text { patient exposed to a study drug for } 1 \text { year, total PEY is the sum of the PEY for each patient, adjusted rate } \\
\text { per } 100 \text { PEY=(total number of events/total years of exposure) } \times 100 ;{ }^{f}: \text { combined data for death and } \\
\text { discontinuation. }\end{array}$} \\
\hline
\end{tabular}


The long-term safety of pirfenidone was evaluated in an open-label extension study of patients with IPF who completed one of the three phase III clinical trials (RECAP, also known as PIPF-012 (NCT00662038)) [23]. The cumulative total exposure to pirfenidone in 1058 patients was 2482 patient-exposure years (PEY) [24]. Treatment-emergent adverse events (TEAEs) were reported as rates per $100 \mathrm{PEY}$ (equivalent to the frequency at which a physician might expect these TEAEs to occur if 100 patients with IPF were followed for 1 year). In RECAP, the most frequent TEAEs were IPF (22.0 per 100 PEY), upper respiratory infection (21.1 per $100 \mathrm{PEY)}$ and bronchitis (19.5 per $100 \mathrm{PEY).} \mathrm{GI} \mathrm{events} \mathrm{(nausea} \mathrm{(17.4} \mathrm{per} 100 \mathrm{PEY}$ ) and diarrhoea (14.4 per $100 \mathrm{PEY})$ ), fatigue (10.1 per $100 \mathrm{PEY})$, cough (17.6 per $100 \mathrm{PEY})$ and dyspnoea (16.9 per $100 \mathrm{PEY}$ ) were also reported. Of note, no new safety signals were identified.

An integrated safety analysis of a large $(n=1299)$ and well-defined cohort of patients with IPF, from an expanded data set of five clinical trials (CAPACITY 004/006, ASCEND, RECAP and PIPF-002 (NCT00080223; a USA-only phase II, multicentre, open-label study of pirfenidone in patients with IPF or secondary pulmonary fibrosis)), demonstrated that pirfenidone was well tolerated and reinforced the safety profile previously characterised in the phase III trials [20]. These patients were treated for up to 9.9 years, with a cumulative total exposure to pirfenidone of 3610 PEY. Nausea (26.0 per 100 PEY), diarrhoea (20.1 per $100 \mathrm{PEY}$ ), fatigue (16.0 per $100 \mathrm{PEY}$ ) and rash (16.2 per $100 \mathrm{PEY})$ were the most common reported AEs in this analysis. Most AEs occurred within the first 6 months of treatment and were generally mild to moderate in severity. The incidence of GI- and skin-related AEs declined after the first 6-month period. Most cases were reversible or manageable by different strategies. A small number of patients $(<4 \%)$ exhibited elevations in aminotransferase levels, which tended to occur in the first 6 months of treatment and were transient and reversible with dose modification or discontinuation.

Earlier phase II trials of pirfenidone as combination therapy or in other ILDs have also shown a similar safety profile. In PANORAMA, a randomised, double-blind, placebo-controlled phase II European study that assessed the safety and tolerability of oral $N$-acetylcysteine (NAC) combined with pirfenidone in patients with IPF ( $n=60$, pirfenidone plus NAC), the addition of NAC did not significantly affect the safety profile of pirfenidone; however, an increased incidence of photosensitivity in the pirfenidone plus NAC versus pirfenidone plus placebo group was observed (13\% versus $2 \%$ ), which could not be explained by additional analyses [25]. The most frequent other TEAEs in the pirfenidone plus NAC group were cough (13\%), nasopharyngitis (12\%), diarrhoea (10\%), nausea (7\%), dyspnoea (7\%) and bronchitis $(7 \%)$. In the group using additional NAC, a more rapid disease progression (as measured by FVC) was observed and the authors conclude that addition of NAC to pirfenidone is unlikely to be beneficial in IPF function [25].

In the phase II, open-label trial that assessed the safety and tolerability of pirfenidone in patients with scleroderma-associated ILD (SSc-ILD) (LOTUSS; NCT01933334), the most commonly reported TEAEs (nausea, headache and fatigue) were consistent with those observed in the pirfenidone phase III trials [26]. The SSc-ILD population is of particular interest for understanding how to manage pirfenidone-related AEs, because this group may be more susceptible to certain AEs and tolerability issues, particularly GI, skin and liver, due to the involvement of these organs in their disease [27, 28].

This safety and tolerability profile of pirfenidone observed in these large, randomised clinical trials has been supported by extensive real-world data and post-marketing experiences. The patient populations in these real-world studies shared similar baseline characteristics with those in the pirfenidone clinical trials. In the expanded access programme (EAP; NCT02141087), a multicentre treatment protocol at 92 sites in the USA that allowed patients with IPF to access pirfenidone before approval by the US Food and Drug Administration, additional safety data were obtained, but no new safety signals were identified [21]. Adverse drug reactions (ADRs; defined by the study investigator as causally linked to drug use; 419.6 per 100 PEY) were reported in $64.9 \%$ of patients $(n=1620)$. The most common ADRs were consistent with the known GI- and skin-related AEs observed in the phase III clinical trials, including nausea (62.1 per $100 \mathrm{PEY}$ ), fatigue (52.4 per $100 \mathrm{PEY}$ ), diarrhoea (27.6 per $100 \mathrm{PEY)}$ and rash (19.6 per $100 \mathrm{PEY})$. Similar ADRs were the most common that led to discontinuation: nausea (6.2 per $100 \mathrm{PEY})$, fatigue (4.4 per $100 \mathrm{PEY})$ and rash (3.2 per $100 \mathrm{PEY})$.

Interim analyses from PASSPORT ( $\mathrm{n}=1009)$, a safety registry study initiated in Europe in 2011, indicated that the safety profile of pirfenidone in routine clinical practice was consistent with that seen in the phase III trials, with no new or unexpected safety signals $[22,29]$. The most common ADRs were nausea $(20.4$ per $100 \mathrm{PEY})$ and fatigue (17.9 per $100 \mathrm{PEY})$. These differences in the incidences of AEs and number of discontinuations observed in the pirfenidone clinical trials versus the registries may be driven by the longer duration of observation, selection/survivor bias, under-reporting in more real-world settings and/or the higher frequency of clinical trial visits and safety assessments in the trial population. 
Prevention and management of common pirfenidone-related AEs

GI- and skin-related AEs were the most common AEs caused by pirfenidone treatment. GI events, such as nausea, vomiting and diarrhoea, typically occur early in the course of pirfenidone treatment and decline after the first 6 months, whereas skin-related AEs, such as rash and photosensitivity, exhibit more variability in their time course $[16,30]$. The median time to any first $\mathrm{AE}$ in the pooled pirfenidone phase III trials was 15.0 days (interquartile range (IQR) 6.0-49.0 days) [31]. For pirfenidone-related AEs, the median (IQR) time to the first GI, nausea, rash and photosensitivity AEs was 14 (5.0-40.0) days, 16.0 (7.0-62.0) days, 82 (49.0-132.0) days and 90 (48.0-149.0) days, respectively (figure 1) [31]. GI and nausea AEs tended to occur during the peri-titration period and dermatological AEs within the first 2-3 months of treatment.

\section{Dose titrations}

The recommended daily maintenance dose of pirfenidone is $801 \mathrm{mg}$ three times per day $\left(2403 \mathrm{mg} \cdot \mathrm{day}^{-1}\right.$ ) with a 14-day titration period upon treatment initiation (table 2) [8]. However, varying the dose titration schedule by using a slower titration may have been an effective means for mitigating the onset of pirfenidone-related AEs. In LOTUSS, a longer titration schedule (4 weeks versus 2 weeks) was associated with better pirfenidone tolerability (table 2) [26]. The 4-week titration schedule was tested with pirfenidone in this specific population because patients with SSc-ILD may be more susceptible to GI-, skin- and liver-related AEs due to the nature of their disease [27, 28]. Patients in the 2-week titration group had more dose modifications overall compared with the 4-week titration group during the titration period (dose reductions: $21.9 \%$ versus $3.2 \%$, difference $18.7 \%, \mathrm{p}=0.0534$; dose interruptions: $12.5 \%$ versus $0 \%$, difference $12.5 \%, \mathrm{p}=0.1132$ ) as well as more discontinuations due to a TEAE (15.6\% versus $3.2 \%)$. Patients in the 2- and 4-week groups had a similar AE profile, most commonly reporting nausea, headache and fatigue, like patients with IPF; however, more patients experienced a severe TEAE in the 2-week subgroup. These results suggest that titration periods of $>2$ weeks may be associated with a better tolerability profile by preventing AEs and further dose modifications for patients receiving pirfenidone.

\section{Dose modifications}

Dose modifications, including reductions or interruptions, are key strategies for AE management with pirfenidone treatment. In the phase III trials, dose reductions and interruptions occurred in $46 \%$ and $41 \%$ of patients receiving pirfenidone, respectively, with a median duration of 28 days and 14 days, respectively [32]. In the pirfenidone-treated population $(n=623), 32.7 \%$ of patients experienced any dose reduction with a median of 23.5 days (IQR 7-95 days) and 34.3\% of patients experienced any dose interruption, with a median of 17.0 days (IQR $8-43$ days). The majority of dose reductions occurred during the first

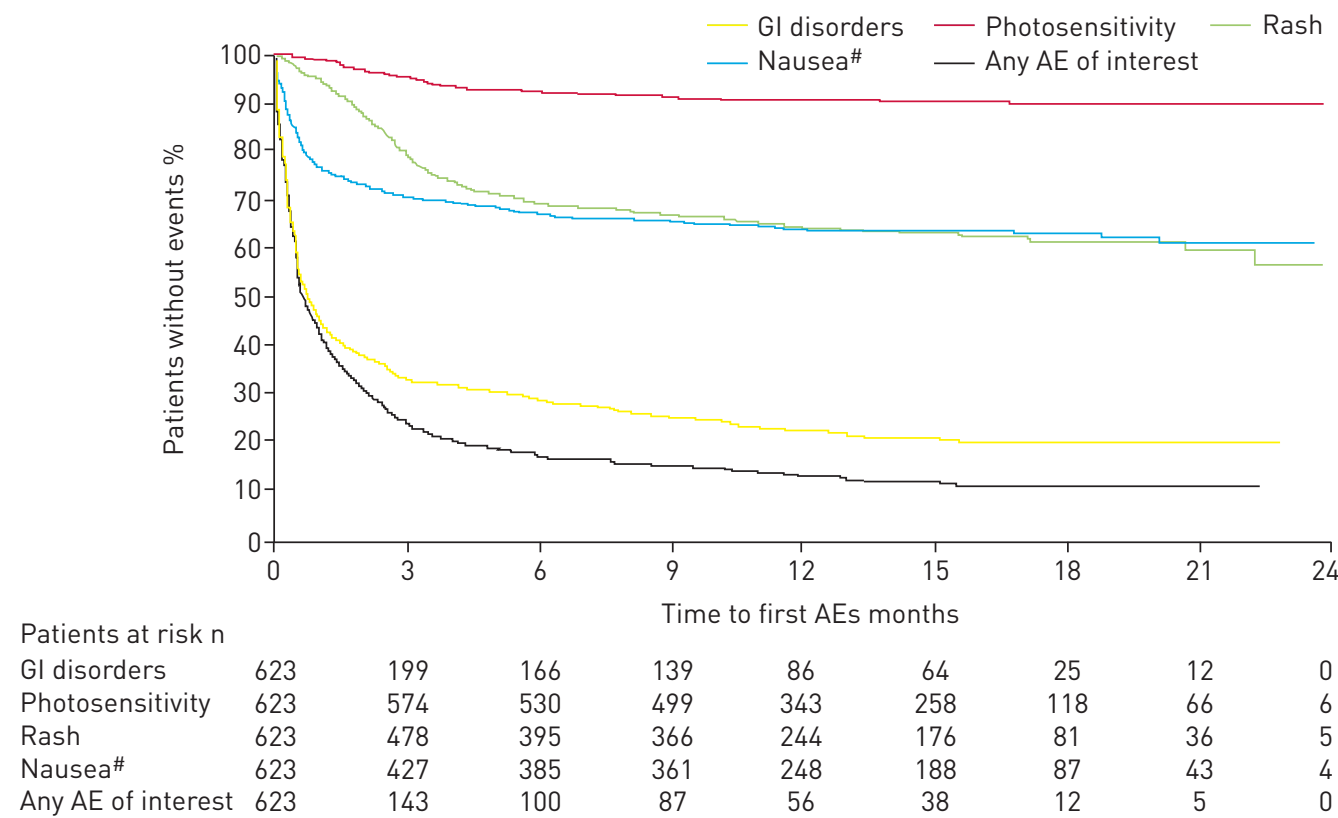

FIGURE 1 Kaplan-Meier analysis of time to first occurrence of a pirfenidone-related adverse event (AE) as measured from randomisation, irrespective of treatment duration, in the pooled phase III clinical trials. GI: gastrointestinal. ": includes all AEs of nausea; these AEs are also included in the group "Gl disorders". 


\begin{tabular}{|c|c|c|c|c|}
\hline & Morning (breakfast) & Afternoon (lunch) & Evening (dinner) & Total pills per day \\
\hline \multicolumn{5}{|c|}{ 2-week titration schedule according to prescribing information [8] } \\
\hline Days $1-7$ & 1 & 1 & 1 & 3 \\
\hline Days 8-14 & 2 & 2 & 2 & 6 \\
\hline Days $\geqslant 15$ & 3 & 3 & 3 & 9 \\
\hline \multicolumn{5}{|c|}{ Modified 4-week titration schedule [26] } \\
\hline Days 1-14 & 1 & 1 & 1 & 3 \\
\hline Days $15-28$ & 2 & 2 & 2 & 6 \\
\hline Days $\geqslant 29$ & 3 & 3 & 3 & 9 \\
\hline
\end{tabular}

Each pill is $267 \mathrm{mg}$. Full dose of pirfenidone is $2403 \mathrm{mg} \cdot$ day $^{-1}$.

6 months of treatment; however, interruptions occurred regularly throughout the year. The median time from start of pirfenidone to the first dose modification for common AEs was $~ 95$ days except for vomiting (28 days) [32]. Although dose modifications with pirfenidone may be required to manage AEs, AEs tended to occur early and were limited in duration, which will allow patients to maintain a high dose intensity.

\section{TABLE 3 Summary of pirfenidone-related adverse event (AE) management strategies}

\section{Pirfenidone-related AEs}

\section{General}

Gastrointestinal

Skin

Liver
Prevention

Slower titration schedule

Taking pirfenidone with a substantial meal, specifically the full dose at the end of a meal or spreading out during a meal

Continuous skin protection with clothing and broad-spectrum SPF 50 sunscreen

Avoid use of other medications associated with phototoxicity

Perform AST, ALT and bilirubin tests before pirfenidone initiation

Monitor at monthly intervals for the first 6 months and then every 3 months thereafter

\section{Management}

Dose reductions

Dose interruptions

Dose reductions/interruptions with a slow titration back to full dose

Reduce the morning dose if nausea is experienced at that time of day

Proton-pump inhibitors

With severe phototoxicity, treat with steroids or silver sulfadiazine

Dose reductions for a rash followed by discontinuation if the rash persists and a slow titration back to full dose

Discontinuation if an allergic reaction to pirfenidone occurs

If AST and ALT elevations ( $>3 \times$ to $\leqslant 5 \times$ ULN) occur without symptoms or hyperbilirubinaemia, the dose may be reduced or interrupted until values return to normal

If AST and ALT elevations ( $>3 \times$ to $\leqslant 5 \times$ ULN) are accompanied by hyperbilirubinaemia, permanently discontinue pirfenidone

If patients exhibit $>5 \times$ ULN, permanently discontinue pirfenidone

\section{Important considerations with drug-drug interactions}

For strong CYP1A2 inhibitors, such as fluvoxamine and enoxacin, pirfenidone should be reduced to $267 \mathrm{mg}$ three times daily $\left(801 \mathrm{mg} \cdot \mathrm{day}^{-1}\right.$ )

For moderate CYP1A2 inhibitors, such as ciprofloxacin at a dosage of $750 \mathrm{mg}$ twice daily, pirfenidone should be reduced to $534 \mathrm{mg}$ three times daily (1602 mg.day ${ }^{-1}$ ) 
Real-world studies have also assessed the use of dose modifications in AE management with pirfenidone. An interim analysis of PASSPORT showed that the rate of discontinuations due to ADRs was lower in patients who had a dose adjustment compared with those who did not (20\% versus 33\%) [22]. In the EAP, of the patients who reached the full daily dose and then had dose modifications/interruptions (and did not discontinue due to an ADR), almost half were able to return to the full dose by the end of the study, suggesting that dose modifications/interruptions may be an effective strategy to allow continuation of treatment with pirfenidone [21].

Smaller, single-centre studies have also documented the benefits of maintaining patients on pirfenidone treatment with dose modifications. In a single-centre, retrospective, observational study of 351 patients who were receiving pirfenidone, $75 \%$ of reported AEs were GI-related, with loss of appetite (17\%) and nausea/vomiting (15\%) being most frequent, similar to what was observed in the phase III trials $[10,11,33]$. The highest number of treatment discontinuations occurred with appetite loss and nausea/vomiting (28 and 22, respectively). These AEs were managed by dose reduction and slower dose titrations. Overall, $30 \%$ of pirfenidone patients had dose modifications and $29 \%$ discontinued permanently due to AEs. Of note, the patients who discontinued treatment had more severe disease by baseline DLCO $(<40 \%)$, indicating that additional strategies may be required for patients who suffer from more severe lung function impairment.

It is important to consider the impact of dose modification on pirfenidone efficacy. A post hoc analysis of the pooled phase III pirfenidone trials assessed the impact of dose reductions and interruptions on the annual rate of FVC decline as a function of dose intensity ( $>90 \%$ of the recommended maintenance dose of $2403 \mathrm{mg} \cdot \mathrm{day}^{-1}$ versus $\leqslant 90 \%$ [32]). A dose intensity of $>90 \%$ was observed in 424 out of 623 pirfenidone-treated and 559 out of 624 placebo-treated patients. The mean \pm SEM difference in annual rate of FVC decline between pirfenidone and placebo over 52 weeks was $105.5 \pm 18.4 \mathrm{~mL}$ for a dose intensity of $>90 \%(\mathrm{p}<0.0001)$ and $102.1 \pm 43.4 \mathrm{~mL}$ for $\leqslant 90 \%(\mathrm{p}=0.0191)$, indicating that the benefit of pirfenidone on FVC was maintained when dose modification was needed. In addition, the dose response with regard to the rate of FVC decline observed in the CAPACITY-004 trial supports the strategy of maintaining the full recommended dose $\left(2403 \mathrm{mg} \cdot \mathrm{day}^{-1}\right)$ of pirfenidone for as long as possible and, when necessary, reducing the dose rather than discontinuing treatment. Patients who received pirfenidone $1197 \mathrm{mg} \cdot \mathrm{day}^{-1}$ had more FVC decline than patients who were receiving the full dose of $2403 \mathrm{mg} \cdot \mathrm{day}^{-1}$, but less than those receiving placebo [10].

\section{GI-related AEs}

An expert panel has recommended stepwise management strategies to prevent and mitigate GI and skin pirfenidone-related AEs and help maintain pirfenidone at an optimal dose for long-term treatment [34]. The preferred approach to managing GI AEs is a temporary dose reduction or interruption, with a slow titration back to the full or previously prescribed dose (table 3 ). The eating habits of the patient should be considered when adjusting the pirfenidone dose. Taking pirfenidone with a substantial amount of food, specifically the full dose at the end of a substantial meal or spreading out the three capsules during the meal, may reduce the rate of pirfenidone absorption and mitigate the onset of GI-related AEs [35-37].

GI and skin pirfenidone-related AEs are manageable with appropriate patient support and education by nurses and physicians. Providing patient education on the timing of these events is a proactive method to improve pirfenidone adherence. One real-world study found that after the occurrence of a GI-related AE within the first 8 weeks and subsequent consultation with a nurse specialist for the following 3 months, pirfenidone adherence was improved by reinforcing necessary management techniques [16].

\section{Skin-related AEs}

One of the most effective management strategies of skin pirfenidone-related AEs is reducing sun exposure through the use of sunscreen and clothing during pirfenidone use [30]. However, if severe phototoxicity occurs, topical treatment with steroids or silver sulfadiazine can alleviate symptoms. The management of skin rashes, including the appropriate diagnosis and control of allergic rashes, is also important and may lead to pirfenidone discontinuation. Similar to GI pirfenidone-related AEs, dose reductions or interruptions can be used (table 3).

\section{Liver function AEs}

Monitoring liver function is also important during pirfenidone treatment [8]. Elevations of aspartate transaminase (AST) and alanine transaminase (ALT) levels to $>3 \times$ the upper limit of normal (ULN) occurred in the phase III trials (3.2\%), which were managed by dose modifications or discontinuation [12]. If AST and ALT elevations ( $>3 \times$ to $\leqslant 5 \times$ ULN) occur without symptoms or hyperbilirubinaemia, the dose may be reduced or interrupted until values return to normal. If the AST and ALT elevations $(>3 \times$ to 
$\leqslant 5 \times$ ULN) are accompanied by hyperbilirubinaemia, pirfenidone should be permanently discontinued. If patients exhibit $>5 \times$ ULN, pirfenidone should be permanently discontinued. These tests should be conducted before initiation of pirfenidone treatment and at monthly intervals for the first 6 months and then monitored every 3 months thereafter (table 3) [8].

\section{Fatigue}

Fatigue is an important pirfenidone-related AE that affects patient quality of life, but is often hard to distinguish from symptoms of the progressive disease [38]. In the pooled phase III trials, fatigue was reported at a rate of 21.2 per $100 \mathrm{PEY}$ [12]. A recent real-world Danish study reported the frequency of fatigue at a higher rate than that in the phase III trials, which had an onset within a few weeks after treatment initiation and was managed by dose modification [39].

\section{AE management in specific patient subgroups \\ Elderly patients}

IPF is strongly associated with age and older patients have a significantly worse prognosis with the disease $[1,2,40,41]$. Elderly patients with IPF also exhibit differences in their rate of decline and have been categorised as either "fit" or "frail"; these patients face specific challenges, such as a more aggressive disease course, lead-time bias with or without delay in diagnosis, and more severe impact of IPF-associated comorbidities [41]. An important consideration for physicians is the number of concomitant medications that elderly patients with IPF receive to manage the comorbidities. Thus, these patients should be carefully monitored for drug-drug interactions, because the patients may be required to take a large amount of oral medications at the same time, which may significantly affect pirfenidone metabolism by inhibiting or inducing hepatic enzyme systems (cytochrome P450 1A2 (CYP1A2), CYP3A4, P-glycoprotein) [41]. For strong CYP1A2 inhibitors such as fluvoxamine and enoxacin, pirfenidone should be reduced to $267 \mathrm{mg}$ three times daily $\left(801 \mathrm{mg} \cdot \mathrm{day}^{-1}\right)$ [8]. For moderate CYP1A2 inhibitors, such as ciprofloxacin at a dosage of $750 \mathrm{mg}$ twice daily, pirfenidone should be reduced to $534 \mathrm{mg}$ three times daily $\left(1602 \mathrm{mg} \cdot \mathrm{day}^{-1}\right)$ [8]. They should also be assessed for GI intolerance, skin reactions and liver enzyme elevations.

A subgroup analysis of patients aged $>65$ years in the two CAPACITY studies showed that there was not an increased incidence of AEs in this population [10]. However, patients aged $\geqslant 80$ years were excluded from the pirfenidone phase III trials, indicating a need for further studies or real-world data for these drugs in this population. More recently in the EAP study, which did not have an upper age limit, a slight age-related increase in the proportion of patients with $\geqslant 1$ ADR was observed, with the highest percentage in patients aged $\geqslant 80$ years. Similarly, the proportion of patients with ADRs leading to dose modification/interruption or discontinuation also increased with increasing age: an ADR leading to dose modification/interruption occurred in $32.7 \%$ of patients aged $\geqslant 80$ years and in $18.0 \%$ of patients aged $<65$ years, while an ADR leading to discontinuation occurred in $20.9 \%$ of patients aged $\geqslant 80$ years and in $7.5 \%$ of patients aged $<65$ years [42]. In a pooled analysis of patients aged $\geqslant 80$ years treated with pirfenidone from RECAP, EAP and an interim PASSPORT analysis $(n=370)$, the safety profile was generally consistent with the overall population from the phase III trials, with the most common ADRs being nausea (33.5 per $100 \mathrm{PEY}$ ), fatigue (26.3 per $100 \mathrm{PEY}$ ) and diarrhoea (18.1 per $100 \mathrm{PEY}$ ) [42]. When patients aged $\geqslant 80$ years were assessed by body mass index, those considered normal/underweight experienced more fatigue, decreased weight, decreased appetite and anorexia, whereas rash was observed equally across body mass index strata. Thus, because this patient population may have a higher number of comorbidities and concomitant medication use, physicians may need to implement more careful management and treatment strategies.

\section{Perioperative management and lung transplant}

A Japanese phase II trial assessed the safety and efficacy of pirfenidone for the prevention of IPF acute exacerbations in patients with lung cancer undergoing pulmonary resection (PEOPLE study) [43]. Only one acute exacerbation was observed in 32 patients with IPF who were treated with pirfenidone and underwent surgery.

Currently there are no established guidelines regarding pirfenidone therapy in perioperative lung transplant [44]. Several case studies of pirfenidone use have been reported. In a single-centre, retrospective study, 12 patients with IPF receiving pirfenidone underwent lung transplant, with all patients discharged from the hospital without wound-healing issues or other complications [45]. Pirfenidone has been successfully administered as a bridge to lung transplant in three patients, with no AEs reported [46]. Following lung transplant, pirfenidone was administered successfully in a woman who experienced progressive allograft dysfunction [47]. In another single-centre, retrospective analysis at a large-volume transplant centre, seven patients receiving pirfenidone underwent lung transplant with no serious 
post-operative AEs observed and no major drug interactions with other perioperative medications [44]. These experiences suggest that pirfenidone can be safely administered before lung transplant with good long-term outcomes.

\section{Patients with severe lung function impairment}

Patients with severe lung function impairment (i.e. FVC $<50 \%$ pred or DLCO $<35 \%$ or $<30 \%$ pred) were excluded from the phase III clinical trials with pirfenidone. Therefore, information about how to manage these patients in clinical practice cannot be obtained from these randomised clinical trials. For 54 patients who had $\mathrm{FVC}<50 \%$ pred at baseline upon entering the open-label extension study RECAP, a higher rate of GI AEs (nausea 37.0\%, vomiting 16.7\%) and a lower rate of skin AEs (rash 13.0\%) was observed compared with the 530 patients who had FVC $\geqslant 50 \%$ pred [48]. However, in an additional analysis of 187 patients who entered RECAP with FVC $<50 \%$ pred or DLCO $<35 \%$ pred, their safety profiles with pirfenidone were similar to those of patients who matched the clinical trial population [49]. In a real-world population of patients who received pirfenidone with $\mathrm{FVC}<50 \%$ pred, a higher rate of nausea was also observed [50].

\section{Patients with comorbidities}

Comorbidities have a significant impact on symptoms and survival associated with IPF. Cardiovascular comorbidities and risk factors are common in IPF [51]. There are no specific warnings or precautions for the use of pirfenidone in patients with cardiovascular comorbidities or in those receiving concomitant anticoagulation therapy, and pharmacodynamics studies have not indicated that pirfenidone prolongs the QTc interval [8]. Of note, the incidence of major cardiac and bleeding AEs was similar over 14 months between pirfenidone and placebo groups in the pooled phase III population [52].

\section{Future research}

Various AE management strategies, including a slower dose titration for initiating treatment, taking pirfenidone with substantial meals, spacing capsules throughout the meal, diet modification, weight-based dosing regimens and dose reductions and interruptions, are all worthy of consideration for future clinical studies. An important question that still remains is understanding how anti-fibrotics, including pirfenidone, affect perioperative safety for patients with IPF who require surgery, including lung transplant. For skin AEs, there are questions about the efficacy of sunscreen in preventing pirfenidone-related rash. Although GI- and skin-related AEs have been commonly addressed in patients with IPF who receive pirfenidone, there is a growing need to understand how to manage fatigue in patients with IPF, whether as a manifestation of the underlying disease or comorbidities or as a side-effect of pirfenidone treatment.

\section{Summary}

The safety data from pirfenidone extension studies, registries and real-world experiences are consistent with pirfenidone's safety profile, as observed in randomised controlled clinical trials. Similar safety profiles have also been observed across different subgroups of interest. GI- and skin-related pirfenidone AEs have exhibited a typical time course that can be mitigated by various prevention and management strategies. Overall, increased vigilance during this time period and dose adjustments are key long-term approaches to managing side-effects and maintaining adherence to pirfenidone therapy (table 3). These dose modifications are flexible and can be optimised for patients based on the severity of observed AEs and responses to changes in dose levels [19]. Physicians must consider the balance between AEs and efficacy in slowing lung function decline when applying these strategies to maintain optimal adherence to pirfenidone therapy [37].

\section{Acknowledgements}

We thank Adefowope Odueyungbo and Philip Hormel (Genentech, Inc., South San Francisco, CA, USA) for statistical analyses. Support for third-party medical writing assistance was provided by Christine Gould (Health Interactions, Inc. San Francisco, CA, USA) and paid for by F. Hoffmann-La Roche Ltd (Basel, Switzerland).

All named authors meet the International Committee of Medical Journal Editors criteria for authorship for this manuscript, take responsibility for the integrity of the work as a whole, and have given final approval to the version to be published.

Support statements declared by individual authors are as follows. L.H. Lancaster was a member of the ASCEND Study Steering Committee; has served as a consultant and on scientific advisory boards for Boehringer Ingelheim, Genentech, InterMune and Veracyte; and participated as a clinical trial investigator for Afferent, Bayer, Boehringer Ingelheim, Celgene, FibroGen, Genentech, Gilead, Stromedix and Veracyte. J.A. de Andrade was a consultant for Boehringer Ingelheim and Genentech/Roche; and has received grants from Boehringer Ingelheim, Genentech/Roche, Global Blood Therapeutics and the National Institutes of Health/National Heart, Lung, and Blood Institute. J.D. Zibrak has received personal fees from Boehringer Ingelheim and Genentech/Roche. M.L. Padilla was an investigator on multicentre studies sponsored by Boehringer Ingelheim and Genentech/Roche, is a faculty member for France Foundation PILOT CME programmes; has served as an advisor and consultant for Boehringer Ingelheim and 
Genentech/Roche; and served as a non-branded speaker for Boehringer Ingelheim and Genentech. C. Albera was a member of the CAPACITY Study Steering Committee; has served on a scientific advisory board for InterMune; and has served as a consultant, steering committee member and speaker for Roche. S.D. Nathan was a member of the ASCEND Study Steering Committee; has served on a scientific advisory board and received research funding from InterMune; has received research funding and served as a consultant for Boehringer Ingelheim, Gilead and Genentech/Roche; is on the speakers' bureau for Genentech/Roche, and his institution has received funding from Genentech/Roche. M.S. Wijsenbeek has received an unrestricted research grant from Intermune/Roche, and speaker and/or advisory board fees from Intermune/Roche, Boehringer Ingelheim and Galapagos. All grants and fees were paid to her institution. J.L. Stauffer and K-U. Kirchgaessler are employees of F. Hoffmann-La Roche Ltd/Genentech, Inc. U. Costabel has served as a member of a study adjudication committee for Gilead; has served as a consultant and speaker for Bayer, Boehringer Ingelheim and InterMune/Roche; has received grants from Boehringer Ingelheim and InterMune; and has served as a consultant for Biogen, Centocor, Gilead, GlaxoSmithKline and UCB Celltech.

\section{References}

1 Fernandez Perez ER, Daniels CE, Schroeder DR, et al. Incidence, prevalence, and clinical course of idiopathic pulmonary fibrosis: a population-based study. Chest 2010; 137: 129-137.

2 Ley B, Collard HR, King TE Jr. Clinical course and prediction of survival in idiopathic pulmonary fibrosis. Am J Respir Crit Care Med 2011; 183: 431-440.

3 Nathan SD, Shlobin OA, Weir N, et al. Long-term course and prognosis of idiopathic pulmonary fibrosis in the new millennium. Chest 2011; 140: 221-229.

4 Raghu G, Collard HR, Egan JJ, et al. An official ATS/ERS/JRS/ALAT statement: idiopathic pulmonary fibrosis: evidence-based guidelines for diagnosis and management. Am J Respir Crit Care Med 2011; 183: 788-824.

5 Ley B, Collard HR. Epidemiology of idiopathic pulmonary fibrosis. Clin Epidemiol 2013; 5: 483-492.

6 Raghu G, Chen SY, Yeh WS, et al. Idiopathic pulmonary fibrosis in US Medicare beneficiaries aged 65 years and older: incidence, prevalence, and survival, 2001-11. Lancet Respir Med 2014; 2: 566-572.

7 Hutchinson J, Fogarty A, Hubbard R, et al. Global incidence and mortality of idiopathic pulmonary fibrosis: a systematic review. Eur Respir J 2015; 46: 795-806.

8 Esbriet (pirfenidone) capsules, for oral use. Package insert. San Francisco, Genentech, Inc., 2017.

9 Raghu G, Rochwerg B, Zhang Y, et al. An official ATS/ERS/JRS/ALAT clinical practice guideline: treatment of idiopathic pulmonary fibrosis. An update of the 2011 clinical practice guideline. Am J Respir Crit Care Med 2015; 192: e3-e19.

10 Noble PW, Albera C, Bradford WZ, et al. Pirfenidone in patients with idiopathic pulmonary fibrosis (CAPACITY): two randomised trials. Lancet 2011; 377: 1760-1769.

11 King TE Jr, Bradford WZ, Castro-Bernardini S, et al. A phase 3 trial of pirfenidone in patients with idiopathic pulmonary fibrosis. N Engl J Med 2014; 370: 2083-2092.

12 Noble PW, Albera C, Bradford WZ, et al. Pirfenidone for idiopathic pulmonary fibrosis: analysis of pooled data from three multinational phase 3 trials. Eur Respir J 2016; 47: 243-253.

13 Nathan SD, Albera C, Bradford WZ, et al. Effect of pirfenidone on mortality: pooled analyses and meta-analyses of clinical trials in idiopathic pulmonary fibrosis. Lancet Respir Med 2017; 5: 33-41.

14 Nathan SD, Albera C, Costabel U, et al. Effect of continued pirfenidone treatment following a $\geqslant 15 \%$ decline in 6-minute walk distance (6MWD) in patients with idiopathic pulmonary fibrosis (IPF): pooled analysis from 3 pivotal studies. Eur Respir J 2016; 48: Suppl. 60, OA1765.

15 King TE Jr, Albera C, Bradford WZ, et al. All-cause mortality rate in patients with idiopathic pulmonary fibrosis. Implications for the design and execution of clinical trials. Am J Respir Crit Care Med 2014; 189: 825-831.

16 Chaudhuri N, Duck A, Frank R, et al. Real world experiences: pirfenidone is well tolerated in patients with idiopathic pulmonary fibrosis. Respir Med 2014; 108: 224-226.

17 Loeh B, Drakopanagiotakis F, Bandelli GP, et al. Intraindividual response to treatment with pirfenidone in idiopathic pulmonary fibrosis. Am J Respir Crit Care Med 2015; 191: 110-113.

18 Oltmanns U, Kahn N, Palmowski K, et al. Pirfenidone in idiopathic pulmonary fibrosis: real-life experience from a German tertiary referral center for interstitial lung diseases. Respiration 2014; 88: 199-207.

19 Wijsenbeek MS, Grutters JC, Wuyts WA. Early experience of pirfenidone in daily clinical practice in Belgium and the Netherlands: a retrospective cohort analysis. Adv Ther 2015; 32: 691-704.

20 Lancaster L, Albera C, Bradford WZ, et al. Safety of pirfenidone in patients with idiopathic pulmonary fibrosis: integrated analysis of cumulative data from 5 clinical trials. BMJ Open Respir Res 2016; 3: e000105.

21 Lancaster L, Morrison L, Auais A, et al. Safety of pirfenidone in patients with idiopathic pulmonary fibrosis in a US expanded access program. Am J Respir Crit Care Med 2016; 193: A2695.

22 Cottin V, Maher TM, Azuma A, et al. Pirfenidone post-authorization safety registry (PASSPORT) update. Eur Respir J 2015; 46: Suppl. 59, OA4500.

23 Costabel U, Albera C, Bradford WZ, et al. Analysis of lung function and survival in RECAP: an open-label extension study of pirfenidone in patients with idiopathic pulmonary fibrosis. Sarcoidosis Vasc Diffuse Lung Dis 2014; 31: 198-205.

24 Costabel U, Albera C, Lancaster LH, et al. Final analysis of RECAP, an open-label extension study of pirfenidone in patients with idiopathic pulmonary fibrosis (IPF). Eur Respir J 2016; 48: Suppl. 60, OA1812.

25 Behr J, Bendstrup E, Crestani B, et al. Safety and tolerability of acetylcysteine and pirfenidone combination therapy in idiopathic pulmonary fibrosis: a randomised, double-blind, placebo-controlled, phase 2 trial. Lancet Respir Med 2016; 4: 445-453.

26 Khanna D, Albera C, Fischer A, et al. An open-label, phase II study of the safety and tolerability of pirfenidone in patients with scleroderma-associated interstitial lung disease: the LOTUSS trial. J Rheumatol 2016; 43: 1672-1679.

27 Savarino E, Furnari M, de Bortoli N, et al. Gastrointestinal involvement in systemic sclerosis. Presse Med 2014; 43: e279-e291.

28 van den Hoogen F, Khanna D, Fransen J, et al. 2013 classification criteria for systemic sclerosis: an American College of Rheumatology/European League against Rheumatism collaborative initiative. Arthritis Rheum 2013; 65: $2737-2747$. 
29 Cottin V, Maher T. Long-term clinical and real-world experience with pirfenidone in the treatment of idiopathic pulmonary fibrosis. Eur Respir Rev 2015; 24: 58-64.

30 Valeyre D, Albera C, Bradford WZ, et al. Comprehensive assessment of the long-term safety of pirfenidone in patients with idiopathic pulmonary fibrosis. Respirology 2014; 19: 740-747.

31 Mason WR, Nathan SD, Zibrak JD, et al. Time-to-event analysis of common adverse events with pirfenidone in patients with IPF - a pooled analysis of three phase III clinical trials. Am J Respir Crit Care Med 2017; 195: A6798.

32 Nathan SD, Lancaster LH, Albera C, et al. Dose modifications and dose intensity during treatment with pirfenidone. Eur Respir J 2016; 48: Suppl. 60, OA1764.

33 Hughes $\mathrm{G}$, Toellner $\mathrm{H}$, Morris $\mathrm{H}$, et al. Real world experiences: pirfenidone and nintedanib are effective and well tolerated treatments for idiopathic pulmonary fibrosis. J Clin Med 2016; 5: E78.

34 Costabel U, Bendstrup E, Cottin V, et al. Pirfenidone in idiopathic pulmonary fibrosis: expert panel discussion on the management of drug-related adverse events. Adv Ther 2014; 31:375-391.

35 Shi S, Wu J, Chen H, et al. Single- and multiple-dose pharmacokinetics of pirfenidone, an antifibrotic agent, in healthy Chinese volunteers. J Clin Pharmacol 2007; 47: 1268-1276.

36 Rubino CM, Bhavnani SM, Ambrose PG, et al. Effect of food and antacids on the pharmacokinetics of pirfenidone in older healthy adults. Pulm Pharmacol Ther 2009; 22: 279-285.

37 King CS, Nathan SD. Practical considerations in the pharmacologic treatment of idiopathic pulmonary fibrosis. Curr Opin Pulm Med 2015; 21: 479-489.

38 The Voice of the Patient: A Series of Reports from the US Food and Drug Administration's (FDA's) Patient-Focused Drug Development Initiative. Idiopathic Pulmonary Fibrosis. Silver Spring, US Food and Drug Administration, 2015. www.fda.gov/downloads/ForIndustry/UserFees/PrescriptionDrugUserFee/UCM440829.pdf

39 Salih GN, Shaker SB, Madsen HD, et al. Pirfenidone treatment in idiopathic pulmonary fibrosis: nationwide Danish results. Eur Clin Respir J 2016; 3: 32608.

40 Ley B, Collard HR. Risk prediction in idiopathic pulmonary fibrosis. Am J Respir Crit Care Med 2012; 185: 6-7.

41 Meyer KC, Danoff SK, Lancaster LH, et al. Management of idiopathic pulmonary fibrosis in the elderly patient: addressing key questions. Chest 2015; 148: 242-252.

42 Lancaster LH, Morrison LD, Auais A, et al. Pirfenidone in patients aged 80 years and older with idiopathic pulmonary fibrosis (IPF): safety findings from pooled trial databases. Am J Respir Crit Care Med 2017; 195: A5385.

43 Iwata T, Yoshino I, Yoshida S, et al. A phase II trial evaluating the efficacy and safety of perioperative pirfenidone for prevention of acute exacerbation of idiopathic pulmonary fibrosis in lung cancer patients undergoing pulmonary resection: West Japan Oncology Group 6711 L (PEOPLE Study). Respir Res 2016; 17: 90.

44 Delanote I, Wuyts WA, Yserbyt J, et al. Safety and efficacy of bridging to lung transplantation with antifibrotic drugs in idiopathic pulmonary fibrosis: a case series. BMC Pulm Med 2016; 16: 156.

45 Mortensen A, Cherrier L, Walia R. Lung transplantation on pirfenidone: a single center experience. J Heart Lung Transplant 2016; 35: Suppl., S302, abstract 833.

46 Riddell P, Minnis P, Ging P, et al. Pirfenidone as a bridge to lung transplantation in patients with progressive IPF Thorax 2014; 69: A183.

47 Ihle F, von Wulffen W, Neurohr C. Pirfenidone: a potential therapy for progressive lung allograft dysfunction? J Heart Lung Transplant 2013; 32: 574-575.

48 Noble PW, Albera C, Kirchgaessler KU, et al. Benefit of treatment with pirfenidone (PFD) persists over time in patients with idiopathic pulmonary fibrosis (IPF) with limited lung function impairment. Eur Respir J 2016; 48: Suppl. 60, OA1809.

49 Costabel U, Albera C, Kirchgaessler K, et al. Analysis of patients with idiopathic pulmonary fibrosis (IPF) with more severe lung function impairment treated with pirfenidone in RECAP. Chest 2016; 150: 537A.

50 Gali JA, Pandya A, Vega-Olivio M, et al. Pirfenidone and nintedanib for pulmonary fibrosis in real-world clinical practice: tolerability and adverse drug reactions. Am J Respir Crit Care Med 2016; 193: A2604.

51 Kreuter M, Ehlers-Tenenbaum S, Palmowski K, et al. Impact of comorbidities on mortality in patients with idiopathic pulmonary fibrosis. PLoS One 2016; 11: e0151425.

52 Glassberg MK, Nathan SD, Lin CY, et al. Cardiovascular events in phase 3 trials of pirfenidone in idiopathic pulmonary fibrosis (IPF). Am J Respir Crit Care Med 2016; 193: A4980. 